\title{
Determine the Optimal Merging Pattern of Toll Plaza \\ Yanhao Wang
}

North China Electric Power University, Beijing, 102206

alanncepu@foxmail.com

Keywords: Traffic capacity; Cellular automaton; Queuing theory; Merging pattern

\begin{abstract}
With the travel rate increasing, Congestion problems have become more prominent. Thus it's essential to enhance traffic capacity, especially for a toll plaza. This paper tries to optimize the merging pattern of vehicles entering into a certain toll plaza .By using cellular automaton and queuing theory finally draw a conclusion of merging pattern. Cellular automaton is used for entering part of toll plaza while queuing theory is used leaving part of toll plaza.
\end{abstract}

\section{Introduction}

As the development of economy, people's travel rate increases, the highway toll port traffic pressure is increasing. By now, there have been a lot of traffic congestion and traffic accidents all over the world, which have serious consequences. As our survey, the shape, size, and merging pattern of a toll station can largely affect its capacity, throughput, incident prevention. For one built-up toll plaza, the thing we can change is to optimize the merging pattern of the toll plaza, and make it achieve the maximum capacity. Considering the heavy traffic harms more, we combine cellular automaton method and queuing theory to simulate traffic flow in a heavy state.

\section{Cellular Automaton Method}

Assumptions. (1).For safety, each vehicle move only one unit of distance to the left side or the right side in the next moment. When entering the toll plaza, vehicles can only change into the adjacent lane.

(2).The same service time for the same kind of tollbooth.

(3).We assume that the size and appearance of each vehicle is the same.

(4).Drivers know the exact number of the waiting vehicles in the adjacent lines when passing the fan-out area, excluding visual illusion.

(5).Drivers obey the traffic rules and drive safely. No accident occurs.

(6).Vehicles will go to the adjacent lanes of their desired tollbooth when entering the fan-out area.

Modeling. (a) the existence of vehicles

1) The number of vehicles

By consulting much information, we know that the relationship between the number of vehicles passing the point and the time they need accords with poisson distribution. With the proceeding of simulation (with time passing by), the number of vehicles appear in simulation space will increase first but decrease later. Especially, electronic toll collection system is the most advanced tolling system. With the development of technology, the proportion of this automatic tolling mode will increase continuously.

2) The occurrence of vehicles

We use function rand in matlab to generate $\mathrm{L}$ random numbers. These random numbers correspond to the serial number of L lanes. Simulative cars run on these lanes randomly.

b) The movement of vehicles

1) In reality, drivers will take control of their own cars depending on different situations. For example, if the car in front brakes and slows down, the car after it will also slow down or change a lane. It's a probability event. We, therefore, set a probability to compare with the random number produced by rand in matlab. This random number ranges from $0-1$. If the random number is less 
than the probability, it will take an action. Or, more than the probability, it will act differently. This program uses this method to achieve the probability event and will also be applied to other probability events in order to simulate real situation.

2) When the driver arrives the fan-out area, they face the problem of choosing the right lanehow to pass the tollbooth as fast as possible. Naturally, drivers will go to the lanes with less vehicles waiting in line. In two-dimensional array of this process, vehicles will decide whether to turn right or left or remain in the original lane by counting the number of vehicles in the fan-out area and the ones in adjacent lanes.

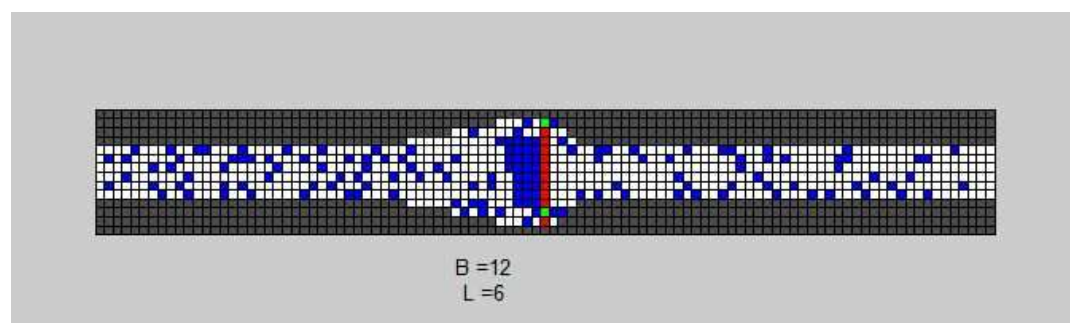

Figure 1. Cellular automaton

c) Charging time of different tollbooths

There are three kinds of tollbooths: conventional tollbooths, exact-change tollbooths, and electronic toll collection booths. Conventional tollbooths and exact-change tollbooths are original methods with longer charging time. Electronic toll collection booths, however, completed the process through radio and computing system. Vehicles needn't stop to pay the toll and can pass the point at relatively faster speed. Consequently, charging time of ETC booths is less than other two ways. And this simulation can embody different charging time of different tollbooths.

Based on assumptions and considerations above, the simulation is quite similar to the real situation, and thus the result of the simulation will be accurate.

\section{Queuing Theory}

Establishment of Model. We didn't consider the traffic jam caused by the increase in number of vehicles in basic model. But in fact, when road load is relatively heavy, vehicles will have to wait in lines to get through the tollbooths. So we can draw a parallel between the volume of congestion and the number of people queuing in the market. We also make an analogy between the conduct of overtaking for the sake of the increase in waiting vehicles to the increase of cashier's efficiency with the increasing number of people. As for queuing system, input procedure is subjected to the poisson process with a $\xi$ parameter. Service time is subjected to a negative exponential distribution with a $\tau$ parameter. Owing to the difference between vehicles queuing situation and market queuing situation, we introduce refining probability. We assume that only vehicles on curve lane have the need to change lanes. The probability of congestion caused by lane-change each time is $\eta$. We assume that each tollbooth is independent and average service rate is $\tau$. When $n>c$, average service rate is $c \tau$. When $n<c$, the average service rate is $n \tau$. Therefore, we can have a general expression of the state transition equation:

$$
\left\{\begin{array}{l}
\tau P_{1}-\xi P_{0}=0 \ldots \ldots(n=0) \\
\xi P_{n-1}+(n+1) \tau P_{n+1}=(\xi+n \tau) P_{n} \ldots \ldots(1 \leq n \leq c) \\
c \tau P_{n+1}+\xi P_{n-1}=(\xi+c \tau) P_{n} \ldots \ldots(n>c)
\end{array}\right.
$$


$P_{n}$ is the probability that the number of customers is $\mathrm{n}$ in steady state, and $P_{0}$ is the probability that no vehicles arrives in $\Delta t$ time interval.

We use queuing theory mainly to solve the problem of highway capacity in heavy traffic. So we focus on the circumstance of $n>c$. We have

$$
\xi P_{n-1}+(c \tau-a(B-L)) P_{n+1}=(\xi+c \tau-a(B-L)) P_{n}
$$

We make $D=c \xi-a B+a L$, and then have

$$
P(z)=P(0)\left(\frac{\frac{D(D+\xi)}{2 D-\xi}}{z-1}+\frac{\frac{D^{2}-\xi^{2}}{2 D-\xi}}{z-\frac{\xi-D}{D}}\right)+P(0)
$$

By inverse transform, we have

$$
P_{n}=P(0) \delta(n)+P(0)\left[\frac{D(\xi+D)}{2 D-\xi}+\left(\frac{\xi-D}{D}\right)^{n} \cdot \frac{D^{2}-\xi^{2}}{2 D-\xi}\right]
$$

$\delta(n)$ in the formula is unit sampling sequence. When $n \neq 0, \delta(n)=0$.

Therefore we can have expectation of geometry distribution, which is the average number of vehicles $(\mathrm{N})$ waiting in line in the system.

$$
N=E(n)=\xi \omega
$$

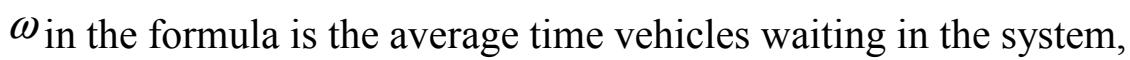

$$
\omega=\frac{N}{\xi} .
$$

Here we still use the number of vehicles passing through the tollbooths in unit time to describe the highway capacity. Considering the possibility of congestion in the fan-out area, we draw out a congestion coefficient $\varphi$ and define it as the ratio of actual traffic capacity in the fan-out area to the designed traffic capacity. The queuing length of in the fan-in area is, then $h_{1}=h \times \varphi$

$$
C_{b}=\frac{L \times \frac{h_{1}}{4}+\varphi(B-L) \times \frac{h_{1}}{4} \times \frac{1}{2}}{\omega}
$$

Substitute $C_{b}$ to the basic programming model, and resolve the formula.

\section{Determine the Solution}

In fan-out area, we use cellular automaton simulation. In fan-in area, we use improved M/M/c queuing theory. Considering different proportions of ETC, MTC and human-staffed tollbooths has a large influence on the conclusion. We set different number for them.

In simulation, we set up 10 tollbooths and 4 lanes. In order to simulate the reality and compare the influence of proportion of different kinds of tollbooths on highway capacity and congestion rate, we set up different proportions of three kinds of tollbooths among these 10 tollbooths. We conduct Simulations as following. 


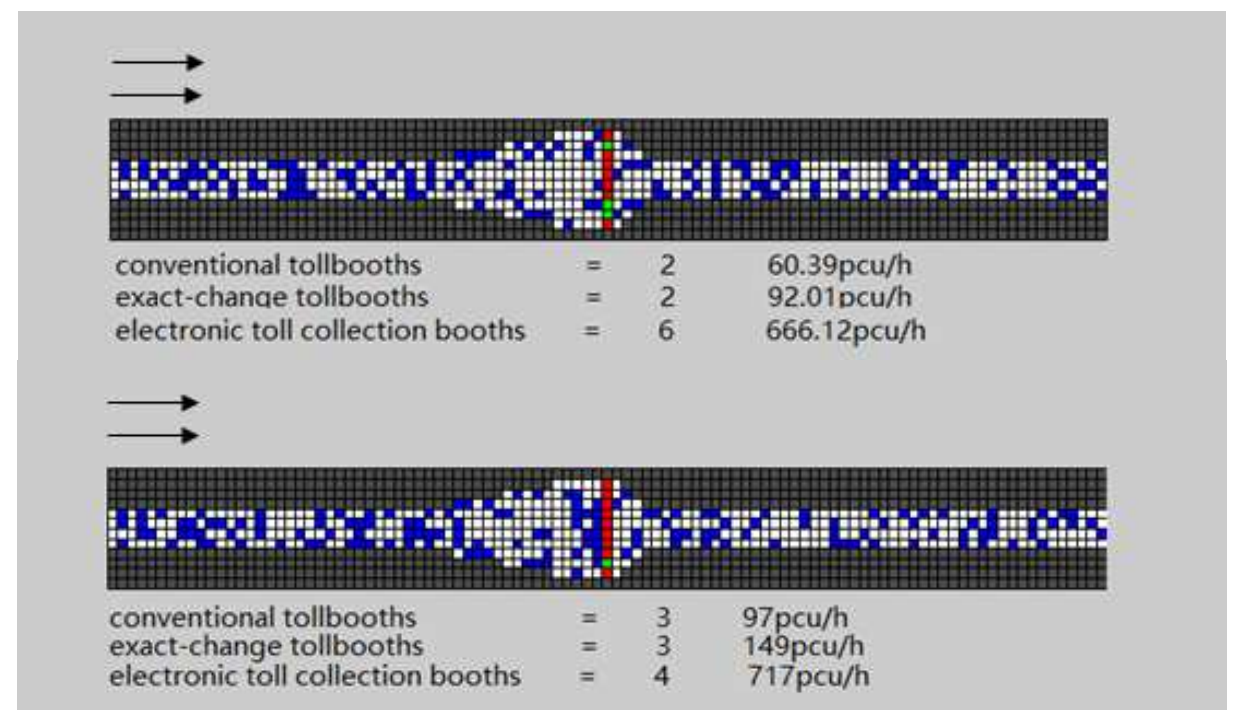

Figure 2.

In queuing theory model, we assume $\lambda=274, \mu=384$,

1) When ETC booths $=2$, MTC booths $=4$, human-staffed tollbooths $=4$ : combine the actual traffic capacity with the designed traffic capacity from the table, then we can have the congestion coefficient, $\varphi=0.5371$, and substitute it into queuing theory model. We get:

$$
P_{n}=0.0253, \omega=4.9012, C_{b}=2924.6
$$

2) When ETC booths=2, MTC booths=4, human-staffed tollbooths=3, in the same way, we can have congestion coefficient $\varphi=0.4007$, and then have:

$$
P_{n}=0.0165, \omega=3.1858, C_{b}=3356.7
$$

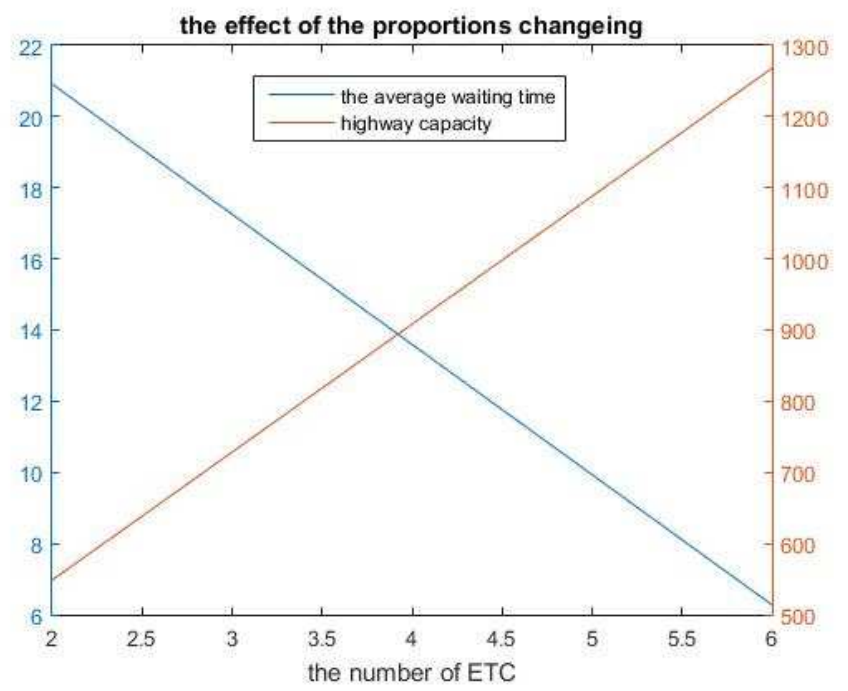

Figure 3.

All in all, heavy traffic should obey the principles that

(a) When entering the fan-in area, the vehicle on the Lth lane don't change the lane

(b) The vehicle can only change into an adjacent lane at each time

(c) Vehicles have the ETC transponder only go through the ETC booths

(d) Vehicles always choose the shortest line to wait

(e) Vehicles without ETC transponder have the probability to drive on the ETC lane. 


\section{References}

[1] Li Xiansheng. Research on the Relation between Road Alignment and Traffic Accidents. [D].Jilin: Jin Lin University.2006.

[2] Zhang Z, Rong J, Zheng H, et al. The Capacity of Expressway Toll-Gate on the Basis of M/G/K Queuing Model in Beijing Region [J]. Highway, 2001.

[3] Wang J, Jia X. The Application of the Theory of Stochastic Service System M/M/C and M/G/K Model [J]. Henan Science, 2010.

[4] Qu Lijie, Zhao Yongjin,Yu Hongbin,Wang Xin,Tao You. Based on the queuing theory model selection of traffic congestion [J]. FuJian Computer, 2013, (10):6-7.

[5] Huang Linna. The queuing theory in the chengdu-chongqing expressway toll station reception the application of the design and management [J]. Standardization of traffic, 2007, (Z1):191-193.

[6] Zhao Xiaoming. Based on cellular automata is compound of high grade highway toll station energy consumption simulation and application [D]. Lanzhou Jiaotong University, 2013.

[7] He L, Wu Y, Zeng X, et al. The design of toll plaza using the combination of modeling and simulation[C]// IEEE International Conference on Grey Systems and Intelligent Services. IEEE, 2011:744-747.

[8] Measuring the efficiency of the outpatient process with queuing theory model [J]. Chinese Journal of Hospital Administration, 2005.

[9] Cheng Y, Hu X H, He X Q. Research on Energy Consumption of Grade highway Traffic System Using Cellular Automata Theory[J]. Automation \& Instrumentation, 2013.

[10] Yang H, Qi Y, Li X, et al. Study on Energy Dissipation of Mixed Traffic Flow Using Cellular Automata[C]// The Twelfth COTA International Conference of Transportation Professionals. 2012:736-743. 